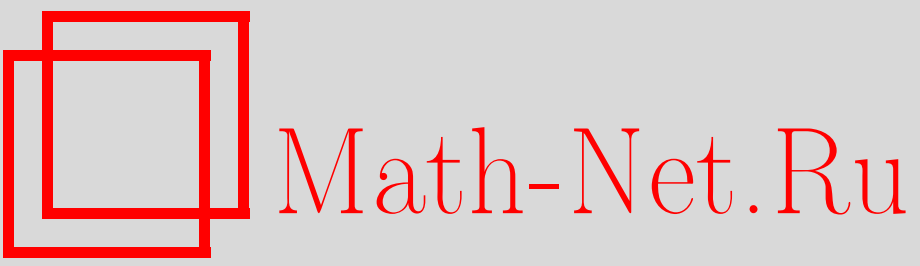

Е. М. Вечтомов, М. Н. Смирнова, Одна двойственность для топологических полуколец непрерывных функций, УМН, 1996, том 51, выпуск 3, 187-188

DOI: https://doi.org/10.4213/rm977

Использование Общероссийского математического портала Math-Net.Ru подразумевает, что вы прочитали и согласны с пользовательским соглашением

http://www.mathnet.ru/rus/agreement

Параметры загрузки:

IP: 54.198 .55 .26

26 апреля 2023 г., 14:43:03 


\title{
ОДНА ДВОЙСТВЕННОСТЬ ДЛЯ ТОПОЛОГИЧЕСКИХ ПОЛУКОЛЕЦ НЕПРЕРЫВНЫХ ФУНКЦИЙ
}

\author{
Е. М. Вечтомов, М. Н. СмирновА
}

Пусть $S$ - топологическое полукольцо. Под полукольцом $S$ понимается система $\langle S,+, \cdot, 0,1\rangle$ такая, что $\langle S,+, 0\rangle$ - коммутативный моноид, $\langle S, \cdot, 1\rangle$ - моноид, тождественно $r(s+t)=r s+r t$, $(r+s) t=r t+s t$ и $0 \cdot s=s \cdot 0=0$, а также $1 \neq 0$. Гомоморфизмы полуколец предполагаются сохраняюшими 0 и 1 . Для топологического пространства $X$ через $C_{p}(X, S)$ обозначим топологическое полукольцо всех непрерывных $S$-значных функций на $X$ относительно поточечных операций и топологии поточечной сходимости. Хаусдорфово пространство $X$ называется $S$-тихоновским, если для любого замкнутого множества $A \subseteq X$ и любой точки $x \in X \backslash A$ найдется $f \in C_{p}(X, S)$, для которой $f(A)=\{0\}$ и $f(x)=1$. Собственный идеал $P$ полукольца называется простым, если для любых элементов $a, b$ полукольца $a b \in P$ влечет $a \in P$ или $b \in P$. Вычисление $\pi_{x}: C_{p}(X, S) \rightarrow S$ в точке $x \in X$ определяется стандартно: $\pi_{x}(f)=f(x)$ для всех $f \in C_{p}(X, S)$. Для $x \in X$ и замкнутого простого идеала $p$ в $S$ положим $P(x, p)=\pi_{x}^{-1}(p)$. Получаем непрерьвный эпиморфизм $\pi_{x}$ и замкнутый простой идеал $P(x, p)$ в $C_{p}(X, S)$.

На топологическом полукольце $S$ рассматриваются условия: а) каждый замкнутый собственный идеал в $S$ содержится в некотором замкнутом простом идеале; b) пересечение любых двух замкнутых простых идеалов в $S$ содержит некоторый замкнутый простой идеал; с) для любъх двух различных әлементов из $S$ существует замкнутый простой идеал в $S$, содержащий ровно один из данных әлементов.

Теорема 1. Пусть $S$ удовлетворяет а), $X$ есть $S$-тихоновское пространство и $P \subseteq C_{p}(X, S)$. Тогда $P$ - замкнутый простой идеал в $C_{p}(X, S)$ в том и только том случае, когда $P=P(x, p)$ для однозначно определенных точки $x \in X$ и замкнутого простого идеала р в $S$.

ДокАЗАтЕльство. Покажем, что произвольньй замкнутый простой идеал $P$ в $C_{p}(X, S)$ имеет вид $P(x, p)$. Если каждый идеал $\pi_{x}(P), x \in X$, всюду плотен в $S$, то, как легко видеть, $P=C_{p}(X, S)$. Поэтому в силу а) $\pi_{x}(P) \subseteq p$ для некоторых $x \in X$ и замкнутого простого идеала $p$ в $S$. Тогда $P \subseteq P(x, p)$. Если также $P \subseteq P(y, q)$ при $y \neq x$, то в силу $S$-тихоновости $X$ найдутся $f \in C_{p}(X, S) \backslash P(x, p)$ и $g \in C_{p}(X, S) \backslash P(y, q)$, для которых $f g=0 \in P$, что невозможно. Следовательно, для всех $y \in X \backslash\{x\}$ идеалы $\pi_{y}(P)$ всюду плотны в $S$.

Пусть, далее, $f \in P, g \in C_{p}(X, S)$ и $g(x)=f(x)$. Докажем, что $g \in P$. Берем конечное множество точек $x_{1}=x, x_{2}, \ldots, x_{n} \in X$ и окрестности $U_{i} \ni g\left(x_{i}\right)$ в $S$. Для каждого индекса $i=2, \ldots, n$ найдется функция $f_{i} \in P$, для которой $f_{i}\left(x_{i}\right) g\left(x_{i}\right) \in U_{i}$ и $f_{i}\left(x_{j}\right)=0$ при $j=1, \ldots, i-1, i+1, \ldots, n$. Возьмем также функцию $\chi$ из $C_{p}(X, S)$, равную 1 в точке $x_{1}$ и 0 в точках $x_{2}, \ldots, x_{n}$. Тогда

$$
h=f \chi+\sum_{i=2}^{n} f_{i} g\left(x_{i}\right) \in P
$$

и $h\left(x_{i}\right) \in U_{i}$ при $i=1,2, \ldots, n$. Таким образом, $g$ принадлежит замкнутому идеалу $P$.

Итак, $P=\pi_{x}^{-1}(p)$ при $p=\pi_{x}(P)$. Поскольку $\pi_{x}$ - открытое гомоморфное отображение на все $S$, то $p$ - замкнутый простой идеал в $S$. Получаем $P=P(x, p)$.

Наряду с $S$ и $X$ возьмем еще топологические полукольцо $T$ и пространство $Y$. Рассмотрим пару $(\alpha, \varphi)$, состоящую из непрерывного гомоморфизма $\alpha: S \rightarrow T$ и непрерывного отображения $\varphi: Y \rightarrow X$. Через $C_{p}(\alpha, \varphi)$ обозначим отображение $C_{p}(X, S) \rightarrow C_{p}(Y, T), C_{p}(\alpha, \varphi)(f)=\alpha$ 。 $f \circ \varphi$ для всех $f \in C_{p}(X, S)$. Ясно, что $C_{p}(\alpha, \varphi)$ - непрерьвный гомоморфизм, сохраняющий константы (функции-константы отождествляем с их значениями).

Теорема 2. Пусть дано отображение $\Gamma: C_{p}(X, S) \rightarrow C_{p}(Y, T)$, причем $S$ удовлетворяет а), $X$ - S-тихоновское и имеет место один из случаев: 1) T обладает свойствами b) и с); 2) $S$ - кольцо, $T$ не имеет делителей нуля и $\{0\}$ замкнуто в $T$. Тогда $\Gamma=C_{p}(\alpha, \varphi)$ для некоторой (единственной) пары $(\alpha, \varphi)$ тогда и только тогда, когда $\Gamma$ - непрерывный гомоморфизм и $\Gamma(S) \subseteq T$. 
ДокАЗАТЕЛЬСтво. Пусть $\Gamma$ - непрерывный гомоморфизм, сохраняющий константы. Сразу получаем непрерывный гомоморфизм $\alpha=\left.\Gamma\right|_{S}$. Определим отображение $\varphi: Y \rightarrow X$. Берем $y \in Y$, произвольный имеющийся в $T$ замкнутьй простой идеал $q$ и $P(y, q)$. По условию а) для $S$ и теореме 1: $\Gamma^{-1}(P(y, q))=P(x, p)$, где $x \in X$ и $p=\alpha^{-1}(q)$. Положим $\varphi(y)=x$. По условию b) для $T$ отображение $\varphi$ задано корректно (в случае 2) условие b) выполняется автоматически). Проверим равенство $\Gamma(f)=\alpha \circ f \circ \varphi$ для произвольной $f \in C_{p}(X, S)$. Возьмем $y \in Y$, $t=\Gamma(f)(y)$ и $s=f(\varphi(y))$. Рассмотрим наши случаи.

Случай 1). Для любого замкнутого простого идеала $q$ в $T$

$$
t \in q \Leftrightarrow \Gamma(f) \in P(y, q) \Leftrightarrow f \in P\left(\varphi(y), \alpha^{-1}(q)\right) \Leftrightarrow s=f(\varphi(y)) \in \alpha^{-1}(q) \Leftrightarrow \alpha(s) \in q .
$$

Значит, $t=\alpha(s)$ на основании условия с) для $T$.

Случай 2). Имеем $f-s \in P\left(\varphi(y), \alpha^{-1}(0)\right)=\Gamma^{-1}(P(y, 0))$. Поэтому $t=\Gamma(f)(y)=\Gamma(s)(y)+$ $\Gamma(f-s)(y)=\alpha(s)$.

Далее, для фиксированного замкнутого простого идеала $q$ в $T$ множества $f^{-1}\left(\alpha^{-1}(q)\right)$, $f \in C_{p}(X, S)$, образуют базу замкнутых множеств $S$-тихоновского пространства $X$. При этом

$$
\varphi^{-1}\left(f^{-1}\left(\alpha^{-1}(q)\right)\right)=(\alpha \circ f \circ \varphi)^{-1}(q)=\Gamma(f)^{-1}(q),
$$

что доказывает непрерывность $\varphi$. Значит, $\Gamma=C_{p}(\alpha, \varphi)$, причем такая пара $(\alpha, \varphi)$ единственна.

Из теоремы 2 вытекает следующая теорема двойственности.

Теорема 3. Категория топологических полуколец $C_{p}(X, S)$ на $S$-тихоновских пространствах $X$ по всевозможным топологическим полукольцам $S$, удовлетворяющим условиям a), b) и с) (или являющимися кольцами без делителей нуля с замкнутыми максимальными идеалами), и их непрерывных гомоморфизмов, сохраняющих константыл, әквивалентна категории соответствующих пар $(S, X)$ с парами $(\alpha, \varphi)$ в качестве морфизмов.

Примеры. 1. Коммутативные полуколца $S$ с тождеством $x^{2}=x$, все идеалы которых замкнуты (ограниченные цепи с интервальной или полуинтервальной топологией) - обладают свойствами а) и с) (свойствами а)-c)). 2 . Пусть $S=\{0,1\}$ - цепь, являющаяся связным двоеточием с замкнутой точкой 0 . Получаем антиэквивалентность категории топологических решеток $C_{p}(X, D)$ и категории всех хаусдорфовых пространств $X .3$. Если дано отделимое полутопологическое поле $S$, то категория топологических колец $C_{p}(X, S)$ двойственна категории всевозможных $S$-тихоновских пространств $X$ (см. [1]). 4. Фиксируя дискретное двухэлементное поле $S$, получаем двойственность между категорией топологических (в поточечной топологии) булевых колец с 1 и категорией всех нульмерных пространств.

ЗАмечАниЕ. Небољшое изменение в доказательстве показьвает, что теорема 1 верна для топологических дистрибутивных решеток $L$, не обязательно ограниченных. Теорема 2 также верна для $L$, толко образ Г должен содержать по крайней мере два элемента.

Из теоремы 3 и замечания следуют соответствующие результаты Капланского, Нагаты, Широты, В.В. Пашенкова, Е.М. Вечтомова по определяемости топологических пространств (см. обзор [2]).

В заключение отметим, что роль топологии поточечной сходимости в тополого-алгебраических исследованиях выпукло показана российскими математиками А.В. Архангельским в [3] и В. В. Пашенковым в [4].

\section{СПИСОК ЛИТЕРАТУРЫ}

[1] Day B. J. // J. Austral. Math. Soc. Ser. A. 1982. V. 32. № 2. P. 171-177. [2] Вечтомов Е. М. // Итоги науки и техн. Алгебра. Топология. Геометрия. Т. 28. М.: ВИНИТИ, 1990. С. 3-46. [3] Архангельский А. В. Топологические пространства функций. М.: Изд-во МГУ, 1989. [4] Пашенков В. В. // УМН. 1987. Т. 42. № 5. С. 79-99. 